\title{
Post-ablative serum thyroglobulin is an independent predictor of recurrence in low-risk differentiated thyroid carcinoma: a 16-year follow-up study
}

Hanna Pelttari, Matti J Välimäki, Eliisa Löyttyniemi ${ }^{1}$ and Camilla Schalin-Jäntti

Division of Endocrinology, Department of Medicine, Helsinki University Central Hospital, PO Box 340, FI-O0290 Helsinki, Finland and ${ }^{1}$ Department of Statistics, University of Turku, FI-20520 Turku, Finland

(Correspondence should be addressed to H Pelttari; Email: hanna.pelttari@kolumbus.fi)

\begin{abstract}
Objective: To study whether post-surgical and/or post-ablative thyroglobulin ( $\mathrm{Tg}$ ) concentrations may serve as independent predictors of disease recurrence in patients treated for TNM stage I or II well-differentiated thyroid carcinoma (WDTC).

Design: An observational retrospective study with a median follow-up of 16 years (range 10-24).

Patients and measurements: Post-operative and post-ablative Tg concentrations, age, tumour size, local infiltration and nodal metastasis at primary surgery as well as disease recurrences and cancer-specific deaths were evaluated in 495 low-risk (TNM stages I and II) patients, the majority of whom had total thyroidectomy and radioactive iodine remnant ablation as initial treatment.

Results: Fifty-one patients (10.3\%) experienced disease recurrence during follow-up. In multiple logistic regression analysis, post-ablative $\mathrm{Tg}$ concentrations (odds ratio $(\mathrm{OR}) 3.72$, confidence interval (CI) 1.71-8.05, $P=0.0009$ ) and local infiltration on primary surgery (OR 2.66, CI 1.03-6.90, $P=0.04$ ) were the only independent predictors of recurrence.

Conclusions: Post-ablative Tg concentration is a strong predictor of disease recurrence in WDTC.
\end{abstract}

European Journal of Endocrinology 163 757-763

\section{Introduction}

Differentiated thyroid carcinoma (DTC) is the most common endocrine malignancy (1) and the incidence has even increased during the past decades $(2,3)$. Most patients are diagnosed at a relatively young age and the prognosis is good, with 5- and 10-year survival rates of $90-95 \%(2,3)$. Despite the low risk of DTCrelated death, disease recurrence rates as high as 35\% have been reported after 40 years of follow-up (2). The number of patients who need monitoring for possible disease recurrence is therefore considerable. While recent guidelines favour lighter follow-up schemes, identification of prognostic risk factors for disease recurrence would help to identify high-risk patients.

Several staging systems have been proposed based on clinical and pathological variables (4-6). However, these systems were created and validated using cancer-specific survival as the outcome and not recurrent disease, which still requires significant intervention. In 2006, the American Thyroid Association recommended the use of pathological TNM (pTNM) staging for all patients with DTC $(7,8)$. This staging system depends heavily on patient age $(<45$ or $\geq 45$ years $)$ and primary tumour size $(\leq 2,>2$ to $\leq 4$ and $>4 \mathrm{~cm}$ ) (6), both of which have been shown to predict cancer-specific survival.

This study therefore aimed to identify factors that predict recurrence after long-term follow-up. We assessed recurrences and cancer-specific deaths in a cohort of 495 patients with well-differentiated thyroid carcinoma, initially treated with both surgery and radioactive iodine remnant ablation (RRA) after a median follow-up of 16 years. We studied possible prognostic factors for disease recurrence in this cohort, including age, gender, primary tumour size, infiltration at primary surgery, nodal metastasis at primary surgery and post-surgical and post-ablative serum thyroglobulin $(\mathrm{Tg})$ concentrations.

\section{Patients and methods}

\section{Patients}

The clinical records of 710 patients (571 females and 139 males) diagnosed with papillary or follicular thyroid carcinoma at the Helsinki University Central Hospital (HUCH) district in a 15-year time period from 1st January 1983 through 31st December 1997 were reviewed. This study material of 495 patients $(70.0 \%$ of all) comprises those differentiated papillary or follicular 
thyroid carcinoma TNM stage I or II patients who were considered to be disease free and in complete remission after primary surgery and RRA therapy. All patients were managed at the HUCH. The follow-up data of this cohort was retrieved by reviewing the clinical records of all patients. A questionnaire regarding the present follow-up of thyroid cancer was additionally sent to patients whose documents showed no recent entries. The 11.6-year follow-up data of the cohort have been described previously (9).

\section{Thyroglobulin}

Data on post-operative and post-ablative serum $\mathrm{Tg}$ concentrations were obtained from the original documents. Post-operative Tg was measured before initiation of thyroxine therapy at the time the patient was evaluated for RRA, e.g. 4-6 weeks after surgery. Post-ablative $\mathrm{Tg}$ measurements in combination with ${ }^{131}$ I whole-body scan (WBS) were performed 4-6 months after the first RRA, under TSH stimulation induced by thyroxin withdrawal.

Until 1989, serum Tg was measured by an RIA kit (Medix Laboratories Ltd, Kauniainen, Finland) with the detection limit of $3 \mu \mathrm{g} / \mathrm{l}$, and after that by an immunometric sandwich assay using two MABs (AutoDelfia, Perkin Elmer, Wallac, Turku, Finland) with the detection limit of $1 \mu \mathrm{g} / \mathrm{l}$. Prevalence of anti$\mathrm{Tg}$ antibodies was evaluated using the recovery method according to the manufacturer's instructions (Delfia Tg (hTG), Perkin Elmer). Briefly, $0.25 \mu \mathrm{g}$ human purified Tg was added to $250 \mu \mathrm{l}$ of each patient's sera in $25 \mu \mathrm{l}$ buffer and mixed well. Tg was measured from serum with and without added $\mathrm{Tg}$ and the recovery was calculated. A recovery of $\geq 80 \%$ was considered normal and excluded the presence of anti-Tg-antibodies. The analytical sensitivity for the $\mathrm{Tg}$ measured was $0.2 \mu \mathrm{g} / \mathrm{l}$.

\section{Study design}

Disease recurrences and cancer-related deaths were recorded and possible predictive variables for disease recurrence were evaluated. A disease recurrence was defined as new evidence of disease after a disease-free period of 12 months or more. It included all sites of disease reported and confirmed either by imaging or by surgery. Disease free was defined as undetectable Tg, no clinical presentation of disease and no radiological evidence of disease. Clinical and histopathological variables included tumour histology, size, presence of nodal metastasis, local infiltration and mode and completeness of surgery (estimated by the performing surgeon) and patient age and gender. Local infiltration was defined as macroscopic adherence of the tumour to the adjoining muscle or soft tissue or microscopic invasion of the adjoining tissue. Biologic variables included $\mathrm{Tg}$ concentrations measured after surgery and after RRA and measurement of Tg antibodies.

\section{Statistical analysis}

The relationship between recurrence and explanatory factors (age, gender, detectable Tg after primary surgery, detectable Tg after RRA, post-operative non-detectable $\mathrm{Tg}$ in the presence of $\mathrm{Tg}$ antibodies, size $(\mathrm{cm})$, follicular histology, total thyroidectomy, complete surgery, nodal metastasis in primary surgery, local infiltration in primary surgery and RRA given) was first examined using simple logistic regression for each factor separately. A multiple logistic regression model including all significant factors in the first analysis was then created. The 95\% confidence intervals (CI) were programmed to hazard ratios. A $P$ value $<0.05$ was considered statistically significant. All tests were performed as twosided tests. S + software was used for statistical analyses. To compare recurrence-free time between patients with detectable and non-detectable Tg, a Kaplan-Meier plot was drawn and log rank test was performed.

\section{Ethics}

The study was reviewed and approved by the ethical committee at the HUCH. Written, informed consent was obtained from all patients if additional information was needed after their follow-up at the $\mathrm{HUCH}$ had ended.

\section{Results}

\section{Patient characteristics and primary therapy}

The study cohort consisted of 415 (83.8\%) female and $80(16.2 \%)$ male patients. Mean age at diagnosis was 40.6 years. Thirty-one $(6.1 \%)$ tumours were of follicular histology, $461(93.1 \%)$ of papillary and 3 of mucoepitheloid histology. Mean tumour size was 1.8 (range $0.3-6.0) \mathrm{cm}$, with the follicular tumours being larger than the papillary (mean 3.0 vs $1.6 \mathrm{~cm}, P=0.1326$ ). Thirteen patients had a tumour classified as $\mathrm{T} 3$ due to size. Total or near total thyroidectomy was performed as primary treatment in $95 \%$ of the patients. Of these 472 patients, $414(87.7 \%)$ received RRA treatment, and repeat doses were administered if necessary. The patient was considered disease free if the neck ultrasound (US) examination was negative and, after the withdrawal of $\mathrm{T} 4$, $\mathrm{Tg}$ was unmeasurable and there was no uptake on WBS. The median RRA activity administered was 3.7 (range 2.2-25.9) GBq. Local tumour infiltration at surgery was detected in $7.4 \%$ of the cases $(n=37)$. Surgery was complete in all but three cases. Locoregional nodal metastases were present in $12.5 \%$ of the cases. Systematic dissection of the cervical lymph node compartments was not performed but pathologic nodes detected before or during surgery were removed. One patient had evidence of distant metastasis at the time of diagnosis, and she was classified as TNM stage II because of her young age. At the time of the present analysis, each patient had a follow-up of at least 10 years (range 10-24) with a median follow-up of 16 years. 


\section{Disease recurrences}

During the median follow-up of 16 years, 51 patients (10.3\%) experienced a disease recurrence. Five of them had a tumour of follicular histology and 46 of papillary. The duration of the disease-free period after primary treatment, the primary diagnostic tool of the recurrence, localisation of the recurrence and post-ablative Tg concentrations for each patient, is presented in Table 1.
Forty-three of the detected recurrences (84.3\%) occurred within 10 years from diagnosis and initial treatment.

The majority of recurrences were located in the cervical lymph nodes $(94.1 \%)$ and were detected with the neck US examination (54.9\%) and were confirmed histopathologically. The diagnosis of a smaller number of recurrences $(31.4 \%)$ was confirmed with a definite new uptake on diagnostic WBS (Table 1). In two cases,

Table 1 The characteristics of patients with recurrence.

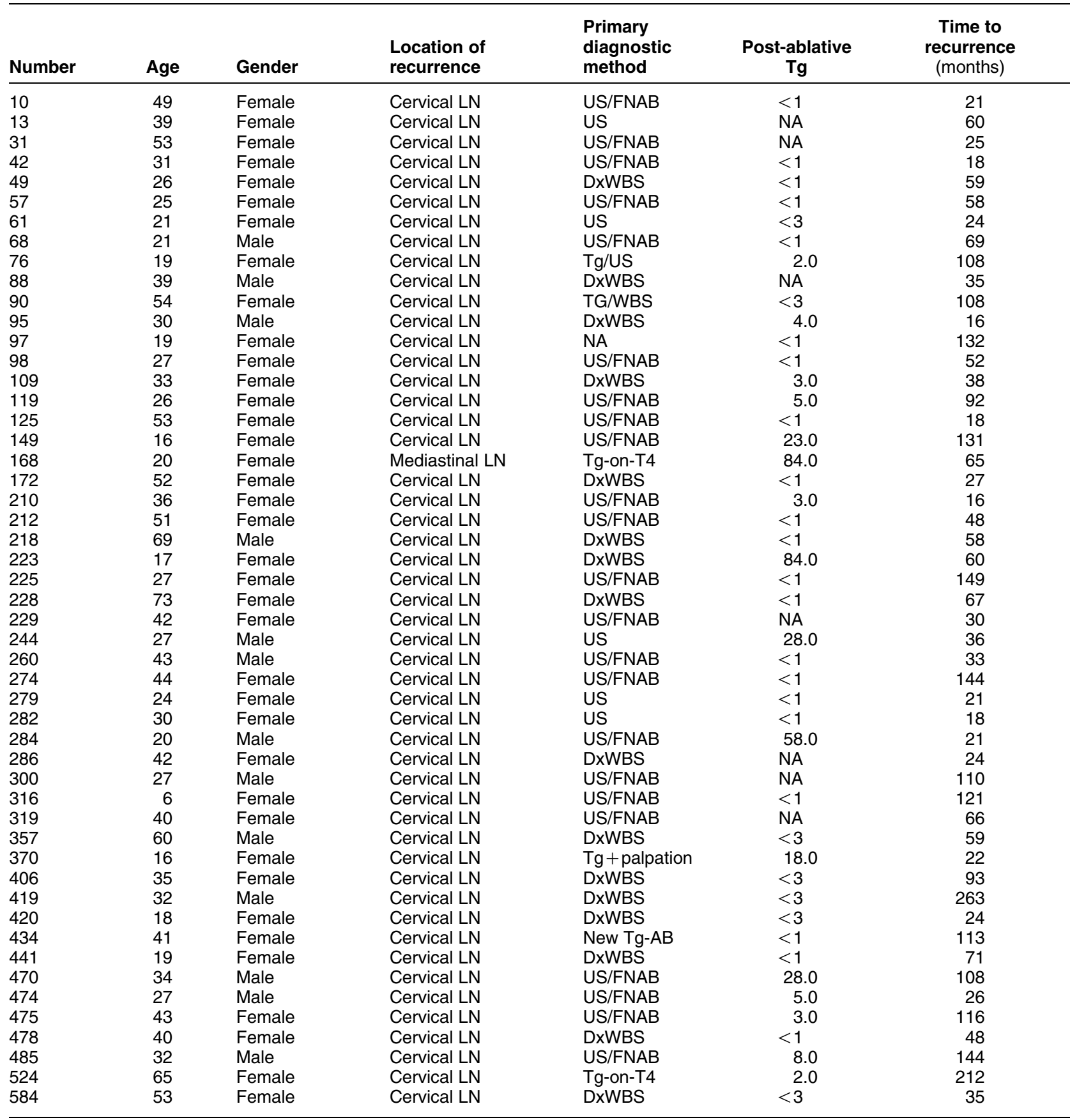

LN, lymph node; US, ultrasound examination; FNAB, fine-needle aspiration biopsy; DxWBS, diagnostic whole body scan with ${ }^{131}$; NA, not available. 
Table 2 Simple logistic analysis of predictive factors for disease recurrence. Thyroglobulin (Tg) concentrations were available for 468 patients and data are expressed as number (percentage) or median (range).

\begin{tabular}{lcrr}
\hline & With recurrence $(n=48)$ & Without recurrence $(n=420)$ & $\boldsymbol{P}$ \\
\hline Age (years) (median with range) & $32(15-73)$ & $40(12-80)$ & 0.0057 \\
Male gender & $13(27.1)$ & $62(14.8 \%)$ & 0.0305 \\
Tg detectable after primary surgery & $34(70.8 \%)$ & $225(53.6 \%)$ & 0.0251 \\
Tg detectable after RRA ablation & $16(36.4 \%)^{\mathrm{a}}$ & $35(9.8 \%)^{\mathrm{b}}$ & \\
Post-ablative non-detectable Tg with Tg antibodies & $6(12.5 \%)$ & $78(18.6 \%)$ & 0.0001 \\
Size (cm) (median with range) & $2.0(0.3-5.0)$ & $1.8(0.1-7.0)$ & 0.3029 \\
Follicular histology & $4(8.3 \%)$ & $24(5.7 \%)$ & 0.1326 \\
TTE & $48(100 \%)$ & $406(96.7 \%)$ & 0.958 \\
Complete surgery & $48(100 \%)$ & $419(99.8 \%)$ & 0.9745 \\
Nodal metastasis in primary surgery & $14(29.2 \%)$ & $47(11.2 \%)$ & 0.0008 \\
Local infiltration in primary surgery & $10(20.8 \%)$ & $25(5.9 \%)$ & 0.0005 \\
RRA given & $46(95.8 \%)$ & $357(85.0 \%)$ & 0.0564 \\
\hline
\end{tabular}

TTE, total or near total thyroidectomy.

$\mathrm{a}_{n=44 .}$.

${ }^{\mathrm{b}} n=357$.

the therapeutic activities of radio-iodine were given because of a significant rise in $\mathrm{Tg}$ on thyroxin without the radiological findings. The recurrence was confirmed with treatment dose WBS. In one case each, recurrence was detected based on increased $\mathrm{Tg}$ concentration on T4 combined with palpable nodes, a suspect US finding and the appearance of $\mathrm{Tg}$ antibodies indicated by declining recovery respectively.

Thyroid carcinoma was registered as cause of death for one patient (data provided by Statistics Finland), who was diagnosed with a metastasised follicular carcinoma at a young age. This case, which initially had been wrongly classified, was reported previously (9).

\section{Post-surgical Tg concentrations and disease recurrence}

Post-surgical $\mathrm{Tg}$ levels were recorded in 468 cases $(94.5 \%)$. After primary total or near total thyroidectomy, 250 cases $(52.9 \%)$ demonstrated post-surgical $\mathrm{Tg}$ levels above the detection limit. Of the 51 patients with disease recurrence, $71 \%(n=34)$ had a post-operative $\mathrm{Tg}$ level above the detection limit as compared with 54\% $(n=225)$ of the 420 patients without recurrence $(P=0.0251$; Table 2). Post-operative $\mathrm{Tg}$ did not correlate with post-operative TSH concentrations $(r=0.056)$. The post-operative median TSH concentrations did not differ between patients with and without recurrence ( 76 and $73 \mathrm{mU} / \mathrm{l}$ respectively, $P=0.39$ ).

\section{Tg concentrations after radio-iodine ablation and disease recurrence}

RRA was performed in 414 cases of the 472 patients who had undergone total or near total thyroidectomy (49 patients with subsequent recurrence and 365 without recurrence). Post-ablative Tg levels were recorded in 401 cases $(96.9 \%)$ and were detectable after the first RRA therapy in 51 cases $(12.7 \%$, median
$10.0 \mu \mathrm{g} / \mathrm{l}$, range 1-500). All patients with detectable $\mathrm{Tg}$ after initial RRA received repeat doses until $\mathrm{Tg}$ became undetectable and there was no evidence of disease on post-therapy WBS. Total ${ }^{131} \mathrm{I}$ activity administered varied between 2.2 and $25.9 \mathrm{GBq}(60-700 \mathrm{mCi})$. After ablation, $\mathrm{Tg}$ was measurable in $36.4 \%$ of patients with subsequent recurrence compared with $9.8 \%$ of patients without recurrence $(P<0.0001$; Table 2$)$. The corresponding post-ablative median TSH concentrations were 44 and $36 \mathrm{mU} / \mathrm{l}$ respectively $(P=0.36)$. Disease recurrence was diagnosed significantly more often in patients with detectable $\mathrm{Tg}$ after initial RRA compared to patients in whom $\mathrm{Tg}$ was undetectable (Fig. 1; $P=0.021$ ).

In seventy-nine cases with undetectable $\mathrm{Tg}$ after RRA, a low recovery $(<80 \%)$ indicated $\mathrm{Tg}$ antibodies. Nine of these patients subsequently developed disease recurrence. There was no statistically significant

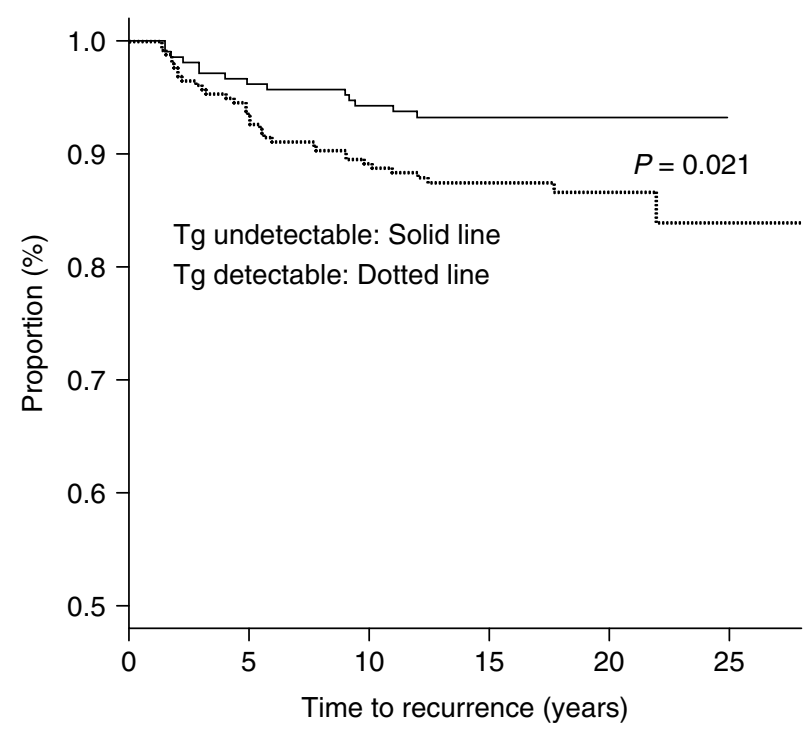

Figure 1 Percentage of recurrence-free patients without $(n=350)$ and with $(n=51)$ detectable $\mathrm{Tg}$ after first ablative radio-iodine treatment as a function of time. 
Table 3 Predictors of recurrence in multiple logistic regression analysis.

\begin{tabular}{lccc}
\hline & Hazard ratio & $\mathbf{9 5 \%} \mathbf{C l}$ & $\boldsymbol{P}$ value \\
\hline Age & 0.978 & $0.978-1.007$ & 0.1369 \\
Post-ablative $\mathrm{Tg}$ & 3.719 & $1.717-8.054$ & 0.0009 \\
Nodal metastasis & 1.489 & $0.629-3.524$ & 0.3651 \\
Local infiltration & 2.66 & $1.025-6.905$ & 0.0444 \\
\hline
\end{tabular}

difference in the prevalence of $\mathrm{Tg}$ antibodies between patients with and those without recurrence $(P=0.3029$; Table 2).

\section{Characteristics of patients with and without recurrence}

Simple logistic analysis of predictive factors for disease recurrence is presented in Table 2. Compared with patients without recurrence, patients who experienced disease recurrence were younger $(P=0.0057)$, more often males $(P=0.035)$ and more often had detectable post-surgical and post-ablative Tg levels $(P=0.0251$ and $P<0.0001$ respectively). Patients with recurrence also had more nodal metastasis $(P=0.0008)$ and local infiltration $(P=0.005)$ at primary surgery.

Of the 37 patients who presented with infiltration at primary surgery, $8(21.6 \%)$ had detectable $\mathrm{Tg}$ concentrations after RRA compared to $9.4 \%$ patients without infiltration. Six of the eight patients $(80 \%)$ were subsequently diagnosed with a recurring carcinoma.

\section{Multiple logistic regression analysis}

Multiple logistic regression analysis including all statistically significant variables in Table 2 revealed that only infiltration at primary surgery (odds ratio (OR) 2.66, CI 1.03-6.90, $P=0.04$ ) and post-ablative detectable $\mathrm{Tg}$ concentrations (OR 3.7, CI 1.71-8.05, $P=0.009)$ were independent predictors of disease recurrence (Table 3).

\section{Discussion}

We present here long-term follow-up data on a cohort of 495 patients at low risk of thyroid cancer-specific death and report the significant association of local infiltration on primary surgery and post-ablative Tg concentrations with disease recurrence after a median follow-up of 16 years. Strengths of this study are a rather great number of patients, all patients uniformly belonging to the favourable TNM classes I-II, and a long follow-up.

During the follow-up, only 1 patient died, but 51 patients $(10.3 \%)$ experienced a recurrence. The recurrence rate thus increased by $2.4 \%$ during this prolonged follow-up of 5 years compared with the recurrence rate of $8.9 \%$ at median follow-up of 11.6 years in the previous report of the same cohort (9). The recurrence rate is steadily approaching that of $14-15 \%$ after 30 years of follow-up in the Mayo Clinic cohort (10). The numbers demonstrate that the follow-up indeed should aim to detect disease recurrences, as the majority of these patients are at low risk of thyroid cancer-specific death. It is therefore important to identify good predictors of well-differentiated DTC recurrences, which could help identify high-risk patients and, on the other hand, enable lighter followup schemes for suitable cases. This would also increase the cost-effectiveness of the surveillance system.

In the revised TNM staging classification of thyroid carcinoma, infiltrative tumours are classified either as $\mathrm{T} 3$ or T4 depending on the grade of invasion (6). In this study, infiltration per se at primary surgery and detectable serum $\mathrm{Tg}$ concentrations after the first RRA (off thyroxine) were the only independent predictors of a later recurrence. Local infiltration is included in nearly all classification systems for predicting cancer-specific death, but only a few studies have examined the possible role of post-surgical or post-ablative $\mathrm{Tg}$ concentrations on disease outcome (11-13). In a study of 268 patients (TNM stages I-III) with a median follow-up of 5.7 years (11), the recurrence rate was $13 \%$ and the authors concluded that post-operative serum $\mathrm{Tg}$ has a complementary role in predicting disease persistence or recurrence. They found a good correlation between post-surgical and post-ablative $\mathrm{Tg}$ concentrations, but did not relate post-ablative concentrations to recurrence rate (11). In the 5.1-year follow-up study of $212 \mathrm{TNM}$ stages I-III patients by Toubeau et al. (12), recurrence rate was $9 \%$ and, in line with this study, post-ablative, but not post-surgical $\mathrm{Tg}$, emerged as a strong predictive factor of disease recurrence, in addition to lymph node invasion. Hall et al. (13) studied 213 DTC patients (TNM stages I-IV) for a median of 47 months, and $12 \%$ experienced recurrence at 11 months. They found that post-surgical $\mathrm{Tg}>20 \mathrm{pmol} / \mathrm{l}$ together with advanced tumour stage was an independent predictor of disease recurrence (13). Taken together, in line with this study, these studies implicate post-surgical/ablative $\mathrm{Tg}$ as a predictor of disease recurrence or progression.

Recently, Sciuto et al. (14) reported that the response to RRA was the strongest predictor of both morbidity and mortality in a cohort of 1503 DTC patients (TNM stages I-IV) with a follow-up of 5 years (14). Although the authors did not report the Tg values in their cohort, a good response to RRA also implies low Tg levels (14). In line with this, recurrence rate $(2.4 \%)$ of 509 patients successfully treated with RRA as evidenced by nondetectable $\mathrm{Tg}$ concentrations and negative WBS 8 months after ablation did not differ between lowand high-risk DTC patients during 12-59 months of follow-up (15). The authors concluded that follow-up should not be based on tumour classification but on the basis of initial treatment response (15). 
The role of RRA in well-differentiated DTC patients at low risk of cancer-specific death has been much debated. An obvious consensus exists that by destroying all the thyroid tissue, RRA not only simplifies the followup based on serum $\mathrm{Tg}$ measurements but also increases its reliability $(7,8,16,17)$. The patients of the present cohort were treated according to a uniform scheme including total or near total thyroidectomy and RRA if not contraindicated. As previously described (9), the cornerstones of the follow-up were neck US examination and fine needle biopsy where indicated and measurements of $\mathrm{Tg}$ concentrations on $\mathrm{T} 4$ therapy. In this uniformly treated patient sample, a detectable post-ablative $\mathrm{Tg}$ concentration measured during thyroxin withdrawal was an independent predictor of later recurrence. The data imply that, in patients treated according to this protocol, more emphasis should be put on the follow-up of patients with detectable postablative $\mathrm{Tg}$ concentrations, while a lighter follow-up scheme probably is indicated in patients with undetectable post-ablative $\mathrm{Tg}$.

That stimulated Tg concentrations measured 6-12 months after initial treatment should be included in the surveillance scheme of DTC patients is strongly emphasised in a number of recent guidelines, not only to control the outcome of primary therapy but also to guide further surveillance $(7,16,18)$. The post-ablative $\mathrm{Tg}$ concentrations predicting recurrence in this study were measured after endogenous TSH stimulation and the results are in line with the present recommendations of the European guidelines (16, 18). The guidelines recommend $\mathrm{Tg}$ measurements stimulated by recombinant human TSH (rhTSH) at 6-12 months after initial RRA. If Tg concentrations are undetectable, follow-up can thereafter be based on yearly evaluation of serum TSH and Tg on T4 treatment with or without neck US examination.

Naturally, the findings of this study are only applicable to patients being treated with RRA. Owing to fear of secondary malignancies, it has been proposed that RRA should be individually tailored (18). It has also been suggested to increase the use of routine prophylactic lymph node dissection and let the results from the node dissection guide the use of RRA (19). The latter suggestions are, however, still under debate (20).

In this study, most of the recurrences and especially the later ones were loco-regional lymph node metastases in the neck. This finding further supports the concept that low-risk patients with DTC can be safely followed up with neck US examination and serum $\mathrm{Tg}$ measurements on $\mathrm{T} 4$, without the use of rhTSH stimulation.

In conclusion, in this prolonged follow-up of 495 DTC patients at low risk of cancer-specific death, recurrence rate was $10.3 \%$ at a median follow-up of 16 years. Post-ablative serum $\mathrm{Tg}$ concentrations and local infiltration at primary surgery were the only independent predictors of recurrence.

\section{Declaration of interest}

The authors declare that there is no conflict of interest that could be perceived as prejudicing the impartiality of the research reported.

\section{Funding}

This study was supported by research funding (Erityisvaltionosuus) from the University Central Hospital in Helsinki.

\section{Acknowledgements}

The Department of Oncology, Helsinki University Central Hospital is acknowledged for permission to review the patient files for postsurgical and post-ablative Tg concentrations. We wish to thank Ms Terttu Säisä for technical assistance.

\section{References}

1 Davies L \& Welch HG. Increasing incidence of thyroid cancer in the United States 1973-2003. Journal of the American Medical Association 2006296 2164-2167. (doi:10.1001/jama.295.18. 2164)

2 Schlumberger MJ. Papillary and follicular thyroid carcinoma. New England Journal of Medicine 1998338 297-306. (doi:10.1056/ NEJM199801293380506)

3 Mazzaferri EL \& Kloos RT. Clinical review 128: current approaches to primary therapy for papillary and follicular thyroid cancer. Journal of Clinical Endocrinology and Metabolism $2001 \mathbf{8 6}$ 1447-1463. (doi:10.1210/jc.86.4.1447)

4 Hay ID, Bergstrahl EJ, Goellner JR, Ebesold JR \& Grant CS. Predicting outcome in papillary thyroid carcinoma; development of a reliable prognostic scoring system in a cohort of 1779 patients surgically treated at one institution during 940 through 1980. Surgery 1993114 1050-1058.

5 Leboulleux S, Rubino C, Baudin E, Caillou B, Hartl DM, Bidart JM, Travagli JP \& Schlumberger M. Prognostic factors for persistent or recurrent disease of papillary thyroid carcinoma with neck lymph node metastasis and/or tumor extension beyond the thyroid capsule at initial diagnosis. Journal of Clinical Endocrinology and Metabolism 200590 5723-5729. (doi:10.1210/jc.2005-0285)

6 TNM classification of malignant tumours. 6th edn. Eds LH Sobin \& C Wittekind. New York: Wiley-Liss, 2002.

7 Cooper DS, Doherty GM, Haugen BR, Kloos RT, Lee SL, Mandel SJ, Mazzaferri EL, McIver B \& Sherman SI. Management guidelines for patients with thyroid nodules and differentiated thyroid cancer. Thyroid 200616 109-142. (doi:10.1089/thy.2006.16.109)

8 Cooper DS, Doherty GM, Haugen BR, Kloos RT, Lee SL, Mandel SJ, Mazzaferri EL, McIver B, Pacini F, Schlumberger M, Sherman SI, Steward DL \& Tutle RM. Revised American Thyroid Association management guidelines for patients with thyroid nodules and differentiated thyroid cancer. Thyroid $2009 \quad 19$ 1167-1214. (doi:10.1089/thy.2009.0110)

9 Pelttari H, Laitinen K, Schalin-Jäntti C \& Välimäki MJ. Long-term outcome of 495 low-risk patients with differentiated thyroid carcinoma followed up with neck ultrasonography and thyroglobulin measurements. Clinical Endocrinology 200869 323-331. (doi:10.1111/j.1365-2265.2008.03217.x)

10 Hay ID. Management of patients with low-risk papillary thyroid carcinoma. Endocrine Practice 200713 521-533.

11 Kim TY, Kim WB, Kim ES, Ryn JS, Yeo JS, Kim SC, Hong SJ \& Shong YK. Serum thyroglobulin levels at the time of ${ }^{131}$ I remnant ablation just after thyroidectomy are useful for early prediction of clinical recurrence in low-risk patients with differentiated thyroid carcinoma. Journal of Clinical Endocrinology and Metabolism 2005 90 1440-1445. (doi:10.1210/jc.2004-1771)

12 Toubeau M, Touzery C, Arveux P, Chaplain G, Vaillant G \& Berriolo A. Predictive value for disease progression of serum 
thyroglobulin levels measured in the postoperative period and after (131)I ablation therapy in patients with differentiated thyroid cancer. Journal of Nuclear Medicine 200445 988-994.

13 Hall FT, Beasley NJ, Eski SJ, Witterick IJ, Walfish PG \& Freeman JL. Predictive value of serum thyroglobulin after surgery for thyroid carcinoma. Laryngoscope $2003 \quad 113$ 77-81. (doi:10.1097/ 00005537-200301000-00014)

14 Sciuto R, Romano L, Rea S, Marandino F, Sperduti I \& Maini L. Natural history and clinical outcome of differentiated thyroid carcinoma: a retrospective analysis of 1503 patients treated at a single institution. Annals of Oncology 200920 1728-1735. (doi:10.1093/annonc/mdp050)

15 Verburg FA, Stokkel MP, Düren C, Verkooijen RB, Mäder U, van Isselt JW, Marlowe RJ, Smit JW, Reiners C \& Luster M. No survival difference after successful (131)I ablation between patients with initially low-risk and high-risk differentiated thyroid cancer European Journal of Nuclear Medicine and Molecular Imaging 2010 37 276-283. (doi:10.1007/s00259-009-1315-6)

16 Schlumberger M, Berg G, Cohen O, Duntas L, Jamar F, Jarzab B, Limbert E, Lind P, Pacini F, Reiners C, Franco FS, Toft A \& Wiersinga WM. Follow-up of low-risk patients with differentiated thyroid carcinoma: a European perspective. European Journal of Endocrinology 2004150 105-112. (doi:10.1530/eje.0.1500105)

17 Sawka AM, Thephamongkhol K, Brouwers M, Thabane L, Browman G \& Gerstein HC. Clinical review 170: a systematic review and meta-analysis of the effectiveness of radioactive iodine remnant ablation for well-differentiated thyroid cancer. Journal of Clinical Endocrinology and Metabolism 200489 3668-3676. (doi:10.1210/jc.2003-031167)

18 Pacini F, Schlumberger M, Dralle H, Elisei R, Smit JWA, Wiersinga W \& The European thyroid Cancer Taskforce. European consensus for the management of patients with differentiated thyroid cancer of the follicular epithelium. European Journal of Endocrinology 2006154 787-803. (doi:10.1530/eje.1.02158)

19 Bonnet S, Martl D, Leboulleux S, Baudin E, Lumbroso JD, Al Ghuzlan A, Chami L, Schlumberger M \& Travagli JP. Prophylactic lymph node dissection for papillary thyroid cancer less than $2 \mathrm{~cm}$ : implications for radioiodine treatment. Journal of Clinical Endocrinology and Metabolism 200994 1162-1167. (doi:10.1210/jc.2008-1931)

20 Mazzaferri EL. A vision for the surgical management of papillary thyroid carcinoma: extensive lymph node compartmental dissections and selective use of radioiodine. Journal of Clinical Endocrinology and Metabolism 200994 1086-1088. (doi:10. 1210/jc.2009-0298)

Received 16 August 2010

Accepted 1 September 2010 